\title{
THE OXYGEN INTAKE AND ENERGY COST OF WALKING BEFORE AND AFTER UNILATERAL HIP REPLACEMENT, WITH SOME OBSERVATIONS ON THE USE OF CRUTCHES
}

\author{
L. G. C. E. Pugh, London, England \\ From the Laboratory for Field Physiology, National Institute for Medical Research, London
}

An opportunity arose of observing the effect of total hip replacement on the energy cost of walking in a patient suffering from post-traumatic monarticular arthritis of the left hip. The patient, aged sixty-two, weighing 78 kilograms and 182 centimetres tall, was a member of the staff of this laboratory and had taken part in exercise experiments over many years. The arthritis developed slowly over six years, becoming rapidly worse during the last year. In November 1971 a Stanmore metal to plastic prosthesis was implanted by $\mathrm{Mr} \mathrm{H}$. B. S. Kemp at the Royal National Orthopaedic Hospital, Stanmore. Before operation the patient was still able to walk two or three miles with the aid of a stick but was much troubled with pain at night. In October 1971 he began to use crutches as a means of taking exercise and learned to walk at up to 8 kilometres per hour with them.

\section{EXPERIMENTAL PROCEDURE AND METHODS}

The oxygen intake was measured by the bag method during sustained walking on the treadmill at a series of speeds up to the patient's maximum. Observations were made on three occasions: 1) one to two weeks before operation; 2) three months after operation; and 3) nine months after operation. The oxygen intake was measured at two to five speeds at each session, depending on the subject's work tolerance at the time. Measurements were made during unassisted walking, walking with a stick held in the right hand, and walking with crutches. Collection of expired gas was started after five minutes at each speed and continued until about 100 litres of gas had been collected. The volume was measured on a dry gas meter, and gas samples were analysed in duplicate on a Lloyd gas analyser.

Energy cost of walking - The net energy cost of walking, which is a measure of efficiency, was calculated as observed oxygen intake minus standing oxygen intake in litres per hour, multiplied by 4.9 , which is the calorie equivalent of oxygen at respiratory exchange ratio of 0.85 , and divided by the speed of walking in kilometres per hour.

\section{RESULTS}

The results are set out in Figures 1 and 2. Each plotted value is a single result. The curves were drawn by eye.

Oxygen intake and speed-Before operation (Fig. 1, Curve 1) the speed of walking was restricted to 3-5 kilometres per hour and the oxygen intake to 0.9-1.2 litres per minute. The maximum stride was 73 centimetres. The use of a stick held in the opposite hand did not affect speed or oxygen intake, although it did extend the distance the subject could walk in a day. The scatter of the results was larger than the scatter in subsequent experiments owing to day-to-day variation in pain and stiffness. Three months after operation (Fig. 1, Curve 2) the subject could walk at speeds up to 7 kilometres per hour with oxygen intake of 1.9 litres per minute, and the oxygen intake at the lower speeds was reduced. Stride length had increased to 115 centimetres. Nine months after operation (Fig. 1, Curve 3) the subject walked at speeds up to 8 kilometres per hour with oxygen intake of 2.4 litres per minute; the oxygen intakes at the lower speeds were identical with those observed in 1955 before the onset of arthritis. There was no further increase in stride. 


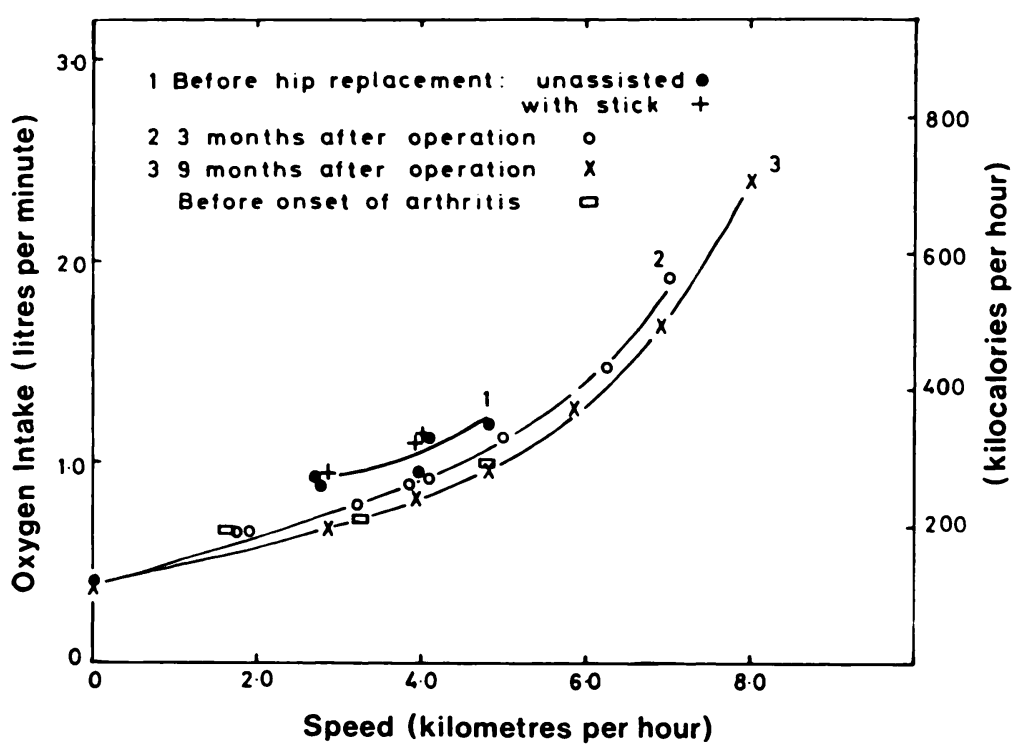

Fig. 1

Relation of oxygen intake and walking speed: before unilateral hip replacement (curve 1); three months after operation (curve 2); nine months after operation (curve 3). Also shown are results of observations made in 1955.

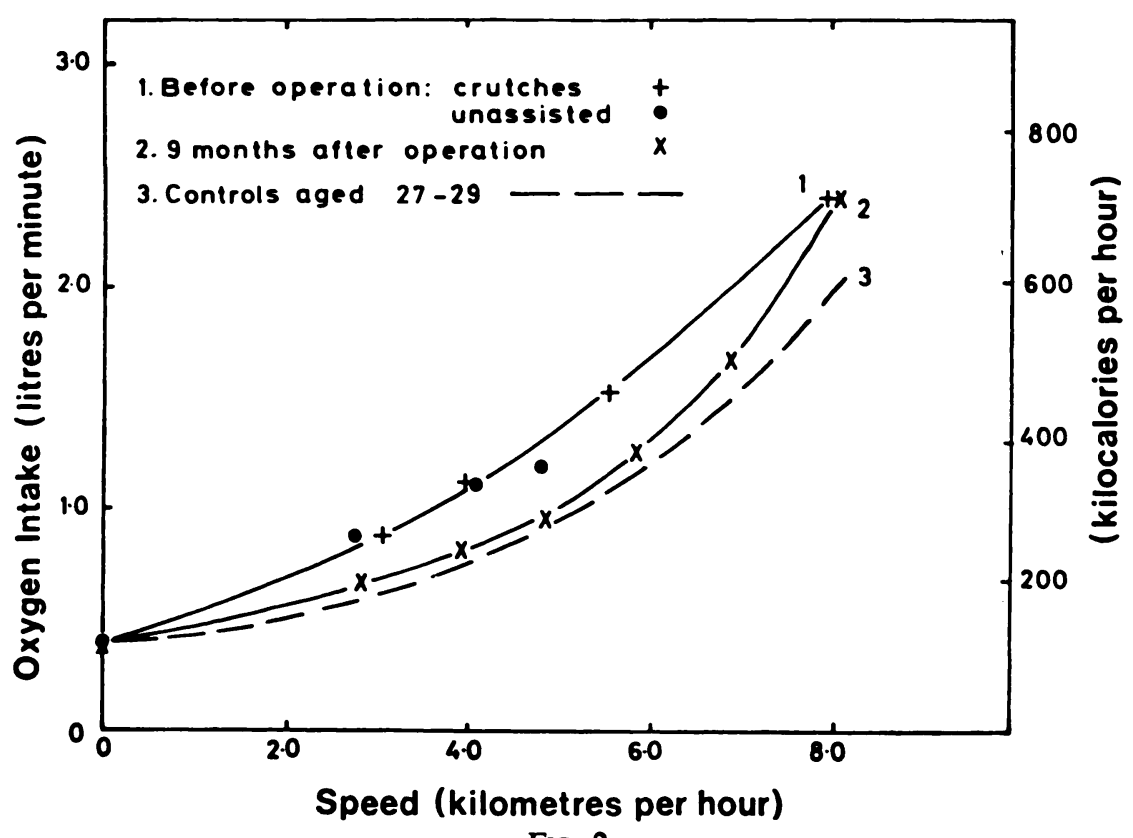

Fig. 2

Effect of using crutches on the relation of oxygen intake and speed of walking. Curve 1, walking with and without crutches before operation; curve 2, unassisted walking nine months after operation; curve 3, control subjects aged 27-29 years. 
Energy cost-The mean energy cost of walking at 3-5 kilometres per hour was 49 kilocalories per kilometre before operation and 31 kilocalories per kilometre after nine months of recovery. The loss of efficiency associated with osteoarthritis was therefore of the order of 58 per cent. Crutches-The use of crutches, like walking with a stick, did not reduce oxygen intake before operation (Fig. 2, curve 1). However, it greatly extended the subject's speed range and oxygen intake, allowing him to exercise at 85 per cent of his maximum oxygen intake, which was determined nine months after operation and found to be $2 \cdot 8$ litres per minute.

The oxygen intake/speed curves for walking with crutches before operation and for unassisted walking nine months after operation are compared in Figure 2 (curves 1 and 2). It is seen that at ordinary walking speeds of 4-6 kilometres per hour, walking with crutches required a higher oxygen intake; but the curve is less steep than that of unassisted walking, and at 8 kilometres per hour the curves intersect. Hence at 8 kilometres the energy cost of using crutches and walking unassisted was the same.

Comparison with control results-Also shown in Figure 2 (curve 3 ) is the oxygen intake/speed curve for three fit male subjects aged twenty-seven to twenty-nine (mean weight 80 kilograms). It is seen that although the oxygen intakes for walking at ordinary speeds were comparable, younger subjects had lower oxygen intakes at high walking speeds. Similarly at 3-5 kilometres per hour the net energy cost of walking was 31 kilocalories per kilometre for the older subject, compared with 28 kilocalories per kilometre for the controls, whereas at 8 kilometres per hour the energy cost was 73 kilocalories per kilometre compared with 57 kilocalories per kilometre respectively.

\section{DISCUSSION}

The above results afford a quantitative expression of impaired function in a case of unilateral osteoarthritis and of the degree of recovery following total hip replacement. The findings illustrate the impairment of speed and mechanical efficiency of walking; they do not, however, illustrate the reduction of distance range or tolerance time, which were equally, if not more, important to the patient. The recovery of efficiency shown by the lower energy cost per kilometre after operation may be of some importance in osteoarthritic patients suffering from associated conditions such as chronic heart and lung disease which restrict oxygen intake, especially for those who are compelled to adopt the device of intermittent movement to overcome restricted oxygen intake. Such patients might expect to find their capacity for continuous movement restored, in addition to relief of pain and stiffness.

We have found no evidence in the literature on the oxygen cost of using crutches. Crutches are not popular with patients and are unsuitable in many occupations. However, for recreational purposes they afford a greatly extended range of distance and allow the subject to work up to his maximum oxygen intake, thus avoiding physical deterioration associated with loss of function due to the arthritis. Walking with crutches was less efficient than normal walking at speeds from 3 to 6 kilometres per hour, but the oxygen intakes were identical at 8 kilometres per hour, which was of interest in that this is the speed at which the oxygen intake/ speed curves for walking and running coincide (Margaria, Cerretelli, Aghemo and Sassi 1963; Menier and Pugh 1968).

The recovery of walking capacity after operation was virtually complete in this patient at ordinary walking speeds. The persistent impairment of the efficiency of walking at high speed is of no practical significance and may be a result of restriction of stride associated with general ageing rather than a residual effect of the osteoarthritis.

There is no information at present on the effect of age on the oxygen intake/speed relation in walking. It is known, however, that age has little effect at slow speeds (Passmore and Durnin 1955). In this subject the relation of oxygen intake and speed in ordinary walking had not changed over seventeen years. The values were, however, somewhat higher than in young control subjects, but still within the normal range. 


\section{SUMMARY}

1. The relation of oxygen intake and walking speed has been investigated in a sixty-twoyear-old patient before and after unilateral hip replacement for osteoarthritis.

2. Before operation walking speed was reduced to three to five kilometres per hour and stride length to 73 centimetres. Oxygen intake was $0.9-1 \cdot 2$ litres per minute and net energy cost 49 kilocalories per kilometre.

3. Nine months after operation the patient could walk at up to 8 kilometres per hour with a stride of 115 centimetres, oxygen intake of 2.4 litres per minute and net energy cost of 34 kilocalories per kilometre. Most of this improvement took place within three months of operation.

4. The oxygen intake of walking at ordinary speeds after full recovery was comparable with that of control subjects aged twenty-seven to twenty-nine years. In very fast walking, however, the oxygen intake was higher than that of the controls, whose oxygen intake was 1.95 litres per minute at 8 kilometres per hour.

5. The use of a stick or crutches did not reduce the oxygen intake in slow walking. However, crutches enabled the subject to walk at over 8 kilometres per hour with oxygen intake of $2 \cdot 4$ litres per minute ( 85 per cent of maximum oxygen intake).

\section{REFERENCES}

Margaria, R., Cerretelli, P., Aghemo, P., and Sassi, G. (1963): Energy Cost of Running. Journal of Applied Physiology, 18, 367.

Menier, D. R., and Pugh, L. G. C. E. (1968): The Relation of Oxygen Intake and Velocity of Walking and Running, in Competition Walkers. Journal of Physiology, 197, 717.

Passmore, R., and Durnin, J. V. G. A. (1955): Human Energy Expenditure. Physiological Revien's, 35, 801. 